\title{
Objective estimation of body condition score by modeling cow body shape from digital images
}

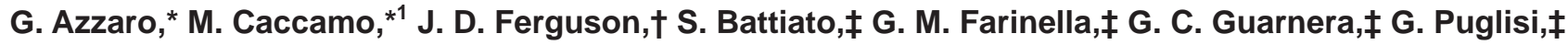 \\ R. Petriglieri,* and G. Licitra*§ \\ *Consorzio Ricerca Filiera Lattiero-Casearia (CoRFiLaC), Regione Siciliana, 97100 Ragusa, Italy \\ †University of Pennsylvania, School of Veterinary Medicine, Kennett Square 19348 \\ łImage Processing Laboratory, University of Catania, 95125 Catania, Italy \\ §Dipartimento di Scienze Agronomiche, Agrochimiche e di Produzioni Animali (D.A.C.P.A.), Agriculture Faculty, University of Catania, 95123 \\ Catania, Italy
}

\section{ABSTRACT}

Body condition score (BCS) is considered an important tool for management of dairy cattle. The feasibility of estimating the BCS from digital images has been demonstrated in recent work. Regression machines have been successfully employed for automatic BCS estimation, taking into account information of the overall shape or information extracted on anatomical points of the shape. Despite the progress in this research area, such studies have not addressed the problem of modeling the shape of cows to build a robust descriptor for automatic BCS estimation. Moreover, a benchmark data set of images meant as a point of reference for quantitative evaluation and comparison of different automatic estimation methods for BCS is lacking. The main objective of this study was to develop a technique that was able to describe the body shape of cows in a reconstructive way. Images, used to build a benchmark data set for developing an automatic system for BCS, were taken using a camera placed above an exit gate from the milking robot. The camera was positioned at $3 \mathrm{~m}$ from the ground and in such a position to capture images of the rear, dorsal pelvic, and loin area of cows. The BCS of each cow was estimated on site by 2 technicians and associated to the cow images. The benchmark data set contained 286 images with associated BCS, anatomical points, and shapes. It was used for quantitative evaluation. A set of example cow body shapes was created. Linear and polynomial kernel principal component analysis was used to reconstruct shapes of cows using a linear combination of basic shapes constructed from the example database. In this manner, a cow's body shape was described by considering her variability from the average shape. The method produced a compact description of the shape to be used for

Received May 24, 2010.

Accepted January 5, 2011.

${ }^{1}$ Corresponding author: caccamo@corfilac.it automatic estimation of BCS. Model validation showed that the polynomial model proposed in this study performs better (error $=0.31$ ) than other state-of-the-art methods in estimating BCS even at the extreme values of BCS scale.

Key words: body condition score, digital imaging, body shape

\section{INTRODUCTION}

Body condition score is widely considered an important tool for management of dairy cattle because it is a simple and repeatable system used to evaluate body fat stores and changes in BCS can be used to estimate cumulative energy balance (Otto et al., 1991; Ferguson et al., 1994). Visual and tactile methods have been used to estimate BCS. Generally, the score range used by dairy management advisors applies a numerical scale, with thin animals receiving lower scores and fat animals receiving higher scores. The system described by Wildman et al. (1982), later modified by Edmonson et al. (1989) and Ferguson et al. (1994) was based on a scale from 1 to 5 , with 1 representing emaciated cows and 5 representing obese cows, without palpating the animal. According to Ferguson et al. (1994), it is possible to separate BCS into 0.25-point increments between scores of 2 to 4 ; however, this degree of resolution may not be possible with BCS of $<2$ and $>4$. It is generally considered that cows with a BCS of $>3.5$ are too fat and that cows having a BCS of $<2.5$ are too thin (Domecq et al., 1997a,b).

Despite the consensus of dairy producers, nutritionists, consultants, and herd managers, on the benefits of the BCS evaluation, less than 5\% of US dairy farms have adopted this practice as an on-farm routine practice (J. D. Ferguson, unpublished data). Many reasons discourage the use of the traditional BCS evaluation techniques: among them is the lack of computerized reports (Ward, 2003), the subjectivity in the judgment that can lead to different scores for the same cow under 
consideration, and the complex as well as time consuming on-farm training of technicians. Furthermore, measurements on a cow must be collected every $30 \mathrm{~d}$ throughout the lactation cycle to have valuable information (Hady et al., 1994), thus increasing the cost and complexity of BCS data.

Interest in collecting information on body condition is increasing for use in selection indices, because selection for production traits has increased reliance of cows on body reserves in early lactation to support milk production (Agnew and Yan, 2000; Coffey et al., 2003). Furthermore, BCS is highly related to reproductive performance: thinner cows experience more reproductive difficulties (Pryce et al., 2000), whereas increasing BCS has positive associations with days to first heat, interval to first service, and conception rate at first service, and negative associations with calving interval and number of services per cow (Agnew and Yan, 2000; Pryce et al., 2000; Dechow et al., 2002; Pryce et al., 2002; Berry et al., 2003; Wall et al. 2003). For this reason, genetic variation in BCS could be included in genetic indexes as an indirect trait for more balanced selection strategies aimed at simultaneously increasing milk production and improving reproductive performance and health (Bastin et al., 2010). The ability to automatically record BCS would increase its use in farm management and enable large volumes of data to be collected for use in national evaluations.

Recent studies have addressed the problem of BCS estimation directly from digital images. Ferguson et al. (2006) assessed the ability to assign a BCS to a dairy cow from digital photographs. In that study, BCS could be assessed by observers from digital photographs or a video taken from the rear of a cow at a 0 to $20^{\circ}$ angle relative to the tail head. A sample size of $30 \%$ was adequate to estimate the mean BCS of a group of cows. Bewley et al. (2008) assessed the feasibility of using digital images to determine BCS using a semiautomatic estimation technique from digital images. They considered a single image of the dorsal view of the cow captured automatically as cows passed through a weigh station and used 23 anatomical points to define the shape of the body of the cow. These points, selected in a manual way, were used to compute 15 angles around the hooks, pins, and tailhead. All identifiable points were used to define and formulate measures describing the cow's contour. Halachmi et al. (2008) tested the hypothesis that the body shape of a fatter cow is rounder than that of a thin cow and, therefore, may better fit a parabolic shape. Images were acquired by means of a thermal camera that allowed a very simple and straightforward shape extraction. The posterior part of the cow was considered and a parabolic fitting was performed. The absolute difference between the real body shape and the fitted parabola was used to estimate BCS for a cow.

Despite the progress in this research area, such studies have not adequately addressed the problem of modeling the shape of a cow's body to build a robust descriptor for automatic BCS estimation. Among the visual cues used by humans, shape provides important information to distinguish between objects of different categories (Belongie et al., 2002) as well as information that is relevant to understand the differences in the appearance of an object within a specific class (Cootes et al., 2001). In computer vision literature, several shape descriptors have been proposed (Persoon and Fu, 1977; Cootes et al., 1992, 2001; Belongie et al., 2002; Di Fabio et al., 2009). More specifically, shape descriptors based on principal component analysis (PCA; Cootes et al., 1992, 2001) are used to consider the different variability of anatomical landmarks with respect to the average shape.

The aim of the present study was to develop a technique to model the body shape of a cow from which learned parameters could be used in BCS estimation. A further objective was to build a benchmark data set useful for dairy cattle research purposes, available through the Internet.

\section{MATERIALS AND METHODS}

\section{System Overview}

A general scheme of the system for semiautomatic evaluation of the BCS from digital images is shown in Figure 1. The system consists of 2 different blocks: training (TB) and employing (EB). The TB is used to learn the parameters of the model exploited to infer the BCS from features extracted on digital images. The parameters are learned by using a set of labeled examples. Once the training is completed, the learned parameters are used in the model to infer the BCS of new samples during the employing phase. Both TB and EB use the same hardware infrastructure (e.g., parameters of digital camera, position of the digital camera).

Each block is composed of different modules organized in a sequential pipeline. The TB is composed of 3 modules as follows: acquisition of training examples, labeling of anatomical features, and learning the BCS model parameters. The acquisition module (AM) is used to acquire images to be used as examples in learning the model parameters. The example cow images generated to be used in the AM should include the range of the number and variety of samples to be acquired. This set of acquired images should be representative of the possible BCS values. During the AM, technicians should evaluate BCS on site of the involved cows to build a 


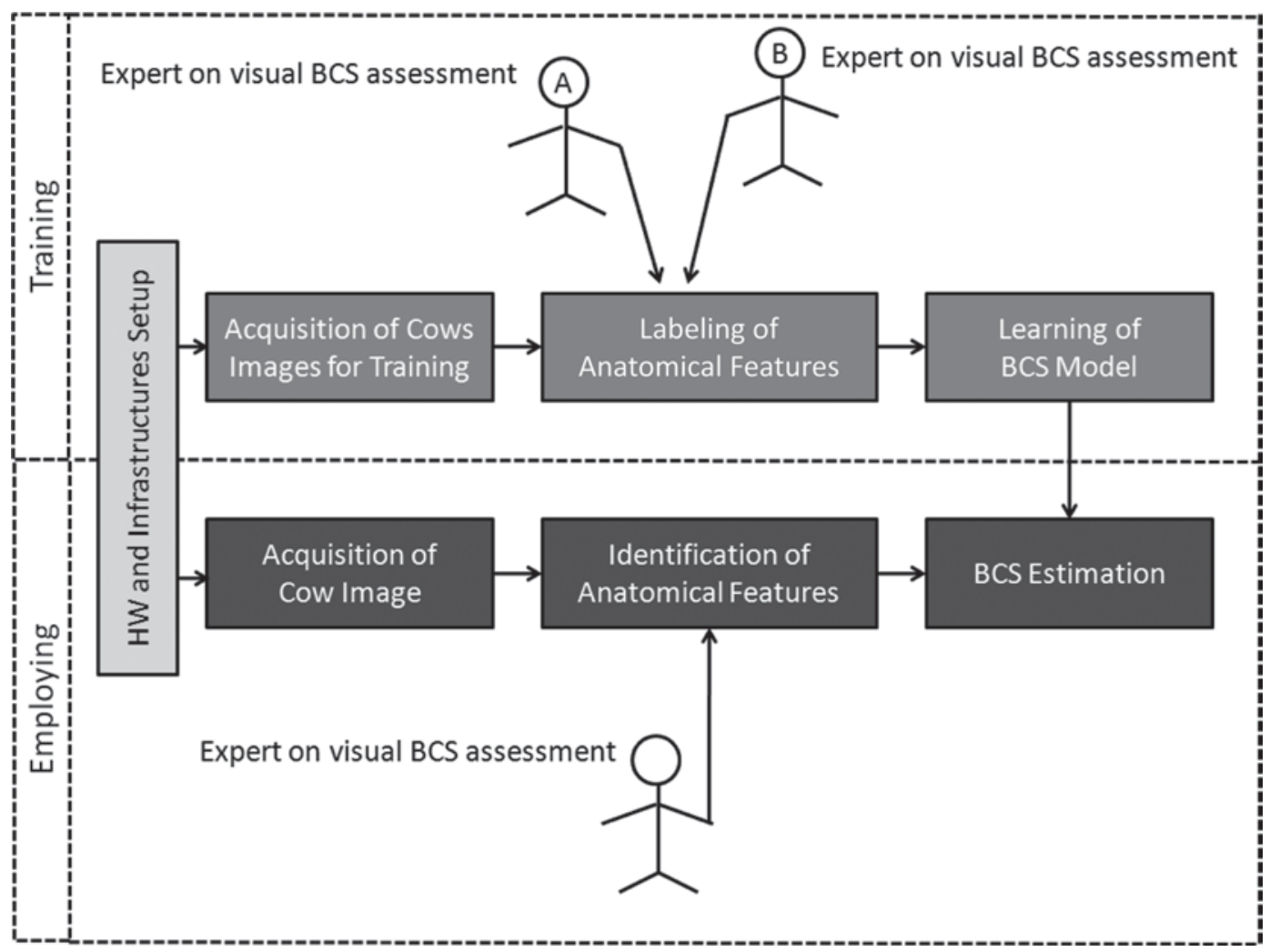

Figure 1. The scheme of a system for semiautomatic estimation of BCS. HW = hardware.

consistent labeled data set containing images with the corresponding BCS. To build a system capable to infer the BCS from anatomical features of cows (e.g., hook angles), the features must be first labeled by experts and then used to learn the parameters of the chosen BCS model. In the labeling module, an expert uses an interface to mark the anatomical features of interest on the acquired digital images. The main labeled components are related to the features that should be taken into account to assess BCS. Several experts and technicians (at least 2) should be used independently in the labeling phase to guarantee that the final labeled data set is not biased by subjectivity of only one technician. The learning module is devoted to establishing the set of parameters involved in the BCS model (e.g., regressor on anatomical points) from the labeled data set. The training block uses the learning and acquisition modules to construct the parameters of a model to estimate the BCS of a cow from the images labeled by the expert(s).

The EB is composed of 3 sequential modules: acquisition of a new unlabeled image of a cow, identification and labeling of anatomical features of the image, and application of the model generated in the learning phase to assign a BCS to the image (and, therefore, cow).

\section{Barn Description and Image Acquisition}

The experiment was performed in a dairy farm located in Comiso (Ragusa, Italy) with 74 first parity lactating Holstein-Friesian cows, housed in a freestall barn with access to a pair of milking robots (DeLaval VMS, Tumba, Sweden).

Images were acquired by means of a standard network digital camera (AXIS 213 PTZ, Lund, Sweden), with pan and tilt functions. This camera could be monitored from the local area network or from the Internet and could switch between color images during daytime, and black/white images in low light conditions (or nighttime) using the built-in infrared lighting. The camera also offered an application programming interface (API) for software integration and its resolution was $704 \times 480$ pixels at up to 30 frames/s for the National Television System Committee (NTSC, the standard to create, transmit, and receive video streams in North America and Japan), and $768 \times 576$ pixels at up to 25 frames/s for the Phase Alternating Line system (PAL, the standard for video transmission used in Europe).

The camera was positioned to capture images dorsally from $3 \mathrm{~m}$ above cows as they passed through the exit gate from the pair of milking robots. The choice of this specific gate guaranteed that all of the lactating 
cows present in that group were examined at least once per day. Cows were constrained briefly at the exit gate for a few seconds, which allowed image capture from a relatively stationary cow.

Images were acquired at an average frame-rate of 12 frames per second from April 4 through May 6, 2009 during 2 time periods: from 1000 to $1400 \mathrm{~h}$ during daylight and from 2000 to $0000 \mathrm{~h}$ to acquire images under 2 different illumination conditions. All images were saved locally and then processed offline. Cows were not restricted based on coat color (cows had variable combinations of white to black markings), coat condition (sleekness of hair coat), or age and size for sample collection.

\section{Cow Identification and Manual Body Condition Scoring}

At the beginning of the acquisition step, 2 technicians were employed to identify the cows at the exit alley of the milking robot. The clocks of both the image acquisition system and the technicians were synchronized. Technicians filled out a report with one record for each cow involved in the experiment. The report contained the cow identification (ID) from a neck collar, BCS assessed by the observer (estimated according to Ferguson et al., 1994), and timestamp. Once the report was completed, the assigned BCS were properly associated to the acquired cow's image by using the timestamp. This procedure produced a data set of 29 images (one for each cow involved in the experiment) labeled with the mean BCS estimated by the 2 technicians.

\section{Image Selection}

During each sample collection period, the image acquisition system gathered a huge amount of data (approximately 172,800 images for each acquisition interval). On average, for each acquisition period, 31 images actually contained a cow in the frame: therefore, the final number of peaks to take into account was set to 40 per acquisition interval in order not to lose useful images. The selection of the images containing cows was a critical issue that could not be addressed manually. To overcome this problem, a 3-step algorithm was developed to select only those images that contained a cow image for analysis. First, a filtering procedure was used employing absolute interframe error $(E)$ or frame difference, which is the analysis of the difference between corresponding pixels in adjacent frames, according to Gonzalez and Woods (2008):

$$
E=\sum_{i=1}^{N} \sum_{j=1}^{M}\left|I_{t}(i, j)-I_{t+1}(i, j)\right|
$$

a)

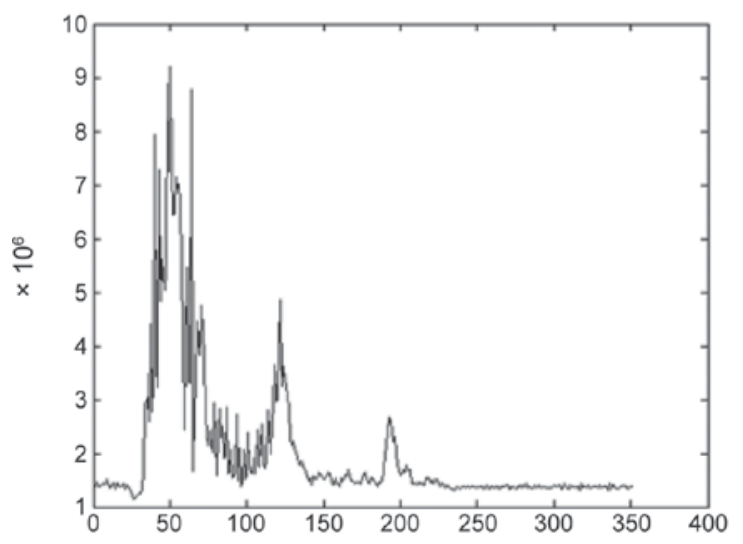

b)


Figure 2. Absolute interframe error relative to a series of selected frames (a), local variance analysis (b), and differences between the identified background frame and the other selected frames (c). The peak indicates the image that differs more with respect to the background (i.e., the image that contains the whole cow). The circle in (b) indicates a plateau in which images are strongly related to consecutive background frames. 
where $I_{t}$ and $I_{t+1}$ are 2 consecutive $M \times N$ frames and $(i$, $j$ ) indicates the pixel position within the frames. When a cow passes through the gate, the absolute interframe error typically has higher values compared with the absolute interframe error comparing frames without a cow (background images). For an acquisition period of $4 \mathrm{~h}$, a peak and valley plot was obtained. Each peak represented an image containing a cow, whereas the valley represented a background image.

After identifying the highest peak images, an automatic process selected a fixed number of images around the peak (200 in our implementation). The image corresponding to the peak might not represent the best image to be used for BCS estimation (e.g., only part of the cow could be visible). The best image to use for BCS had to be selected from the 200 selected images. The mean absolute interframe error (Figure 2a) was then employed as a starting point for local variance analysis from the 200 images (i.e., the variance was computed considering a sliding window of 20 elements). The plateau was strongly related to consecutive background frames (as highlighted in Figure 2b). A background frame $(\mathbf{B g})$ was selected from this uniform region and the difference between all of the selected frames and Bg was computed (Figure 2c). The peak indicated the frame that differed the most with respect to $\mathrm{Bg}$ : the corresponding frame, therefore, probably contained the whole cow.

To cope with motion blur, focus, and other acquisition problems, 5 frames around the identified frame were selected. Finally, the best frame was manually identified among the 5 frames. Human interaction was, therefore, present only at the end of the process and was related to a small subset of frames $(5 \times 40$ per $4 \mathrm{~h}$ of acquisition).

\section{Anatomical Points Labeling}

An ad hoc JAVA application was implemented to label the 23 anatomical points useful for BCS estimation according to Bewley et al. (2008). This software is available, upon registering, for download at http:// www.corfilac.it/bcs/software.html, subject to the general public license (GPL). The graphical user interface is shown in Figure 3. The main window was divided into 4 areas: lower areas show a reference figure and a legend of the key points to locate; upper areas show the image of a cow to be labeled and the labeled point currently identified.

An image is automatically loaded from a user-specified directory and presented in the box in the upper left corner (Figure 3). All 23 anatomical landmarks to be marked are listed in the legend in the order in which they are to be identified. To select a point, the user uses a mouse to point to a region and right clicks on the mouse to record the location. The selection of a point activates 2 buttons (upper right quadrant in Figure 3): the first cancels the point previously identified, whereas the second permits acceptance of the new point identified and enables the user to move forward (labeling errors can be immediately corrected). A counter in the top right box records the anatomical landmarks as they are identified by the user (Figure 3). Once the twentythird anatomical point was confirmed, the software performed a consistency check of the selected points, identifying the most likely errors in their placement order (e.g., points from 1 to 9 should have an increasing abscissa).

\section{Shape Alignment}

To obtain a consistent shape representation, location, scale and rotational effects were filtered out by aligning the corresponding anatomical landmarks of the different involved shapes. The alignment of shapes was carried out by establishing a coordinate reference (position, scale, and rotation, commonly known as pose) to which all shapes referred. The reference anatomical landmarks were the fore ribs, the tail, and the right and left hook, as highlighted in Figure 4a.

Because the anatomical landmarks that define the shapes refer to the image coordinate system, at first, shapes were translated to the origin (Figure $4 \mathrm{~b}$ ). Shapes were then rotated such that the left ileal tuberosity (hook bone) and the right ileal tuberosity (hook bone) had the same horizontal coordinate (Figure 4c). To perform translation and rotation of shapes, the middle point between the left hook bone and the right hook bone was taken into account. The choice of the midpoint between the hook bones provided a fixed point of reference for performing translation and rotation of the body image with respect to this point so that the shapes just differed on scale, which could be scaled to fit in a unit square (Figure 4d). The landmarks with minimum and maximum values of $\mathrm{x}$ coordinates were used to scale the shape with respect to the $\mathrm{x}$-axis, whereas the landmarks with minimum and maximum values of $y$ coordinates were used to scale the shape with respect to the y-axis. The scaling of shapes makes the system invariant to scale. In this way, the system can be used independently from the distance of the camera from the ground floor without running again any learning phase. After alignment, all of the shapes referred to the same coordinate system (centered into the origin) and could be modeled by using the statistics on the 23 anatomical landmarks. 


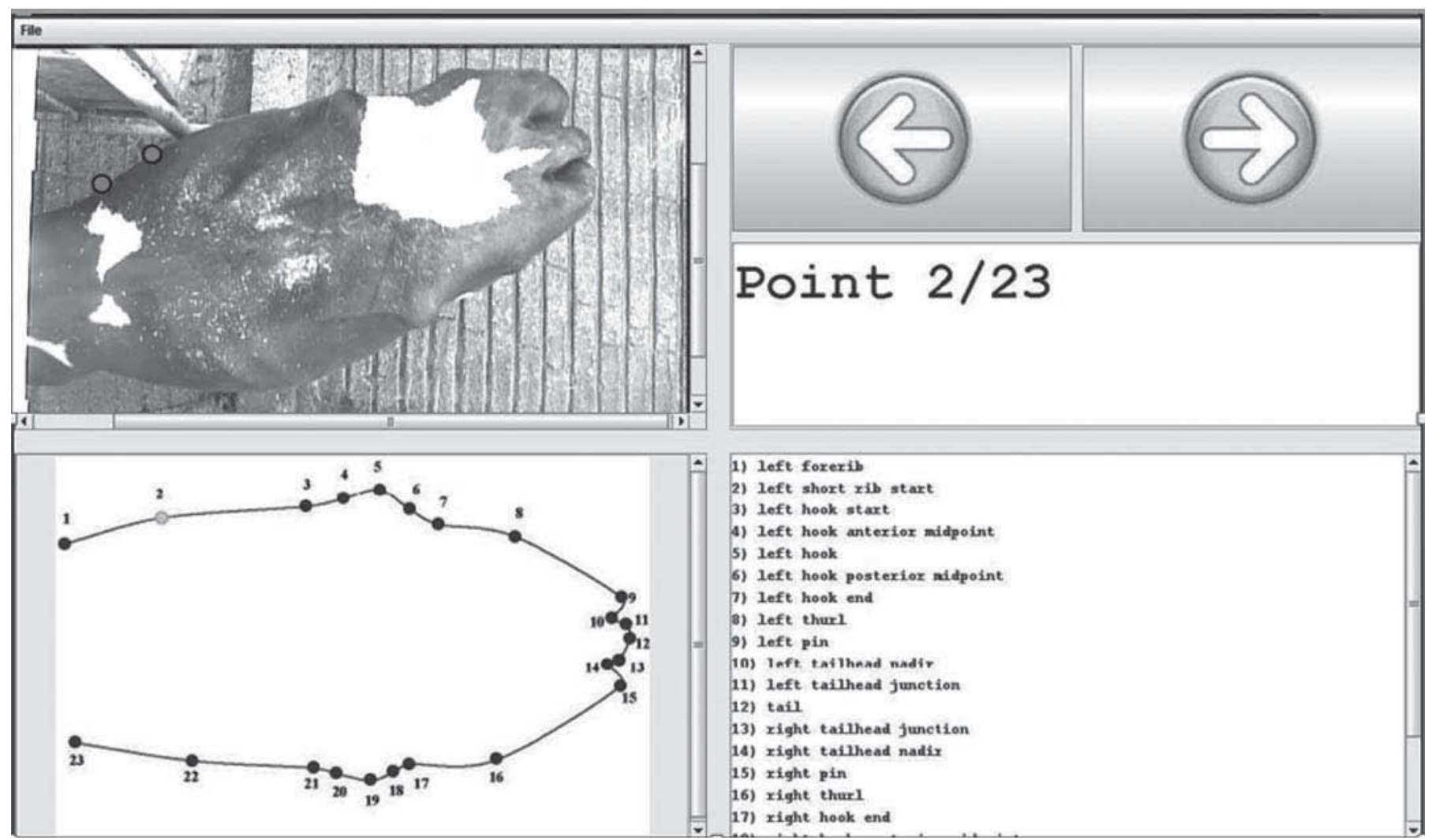

Figure 3. The JAVA interface of the labeling software used to mark the 23 anatomical points.

\section{PCA-Based Shape Descriptor}

We started by considering the following shape definition: shape is all of the geometrical information that remains when location, scale, and rotational effects are filtered out from an object (Dryden and Mardia, 1998). Shapes are typically represented by locating a finite number of landmarks on the outline of an object. The mathematical representation for $n$ landmarks located into the shape of an object is a $2 n$-dimensional column vector:

$$
\begin{gathered}
\mathbf{s}=\left[\mathrm{x}_{1}, \mathrm{x}_{2}, \ldots, \mathrm{x}_{\mathrm{n}}, \mathrm{y}_{1}, \mathrm{y}_{2}, \ldots, \mathrm{y}_{\mathrm{n}}\right]^{\mathrm{T}}= \\
{\left[\mathrm{s}_{1}, \mathrm{~s}_{2}, \ldots, \mathrm{s}_{\mathrm{n}}, \mathrm{s}_{\mathrm{n}+1}, \mathrm{~s}_{\mathrm{n}+2}, \ldots, \mathrm{s}_{2 \mathrm{n}}\right]^{\mathrm{T}},}
\end{gathered}
$$

where $\left(\mathrm{x}_{\mathrm{i}}, \mathrm{y}_{\mathrm{i}}\right)$ for $\mathrm{i}=1, \ldots, \mathrm{n}$, are the coordinates of the $\mathrm{n}$ landmarks and $\mathrm{T}$ indicates the transpose operator of vectors. Let $\mathbf{S}=\left\{\mathbf{s}_{1}, \ldots, \mathbf{s}_{\mathrm{m}}\right\}$, a set of shapes, and $\mathbf{S}^{\prime}=$ $\left\{\mathbf{s}_{1}^{\prime}, \ldots, \mathbf{s}_{\mathrm{m}}{ }_{\mathrm{m}}\right\}$, the set of shapes obtained through the alignment procedure. The mean shape of $\mathbf{S}^{\prime}$ corresponds to the vector that minimizes the sum of the squarederror criterion function with respect to the shapes in $\mathbf{S}^{\prime}$. Hence, the sample mean $\overline{\mathbf{s}}^{\prime}$ is the zero-dimensional descriptor of the data set $\mathbf{S}^{\prime}$ and can be considered as a prototype of the data, in the sense that it is the most similar to all of the samples in the data set, but it does not reveal any of the variability in the data. The modes of variations, the ways in which the points of the shape tend to move with respect to the average shape, can be found by applying PCA to the deviations from the mean $\overline{\mathrm{s}}^{\prime}$ (Cootes et al., 1992; Cootes et al., 2001; Stegmann and Gomez 2002). More specifically, taking into account the $2 n \times 2 n$ covariance matrix $\mathbf{C}$, built taking into account the samples in $\mathbf{S}^{\prime}$, the modes of variation of the points of the shapes are described by the unit eigenvectors of $\mathbf{C}$, such that

$$
\begin{array}{cc}
\mathbf{C} \mathbf{e}_{i}=\lambda_{i} \mathbf{e}_{i} & i=1, \ldots, 2 n \\
\mathbf{e}_{i}^{T} \mathbf{e}_{i}=1 & i=1, \ldots, 2 n,
\end{array}
$$

where $\lambda_{i}$ is the $i$ th eigenvalue of $\mathbf{C}$. The eigenvectors $\mathbf{e}_{i}$ of the covariance matrix corresponding to the largest eigenvalues describe the most significant modes of variations in the variables used to derive the covariance matrix.

\section{Kernel PCA-Based Shape Descriptor}

Kernel PCA has been successfully used for statistical shape analysis (Rathi et al., 2006; Sahbi, 2007). Kernel 




$a$

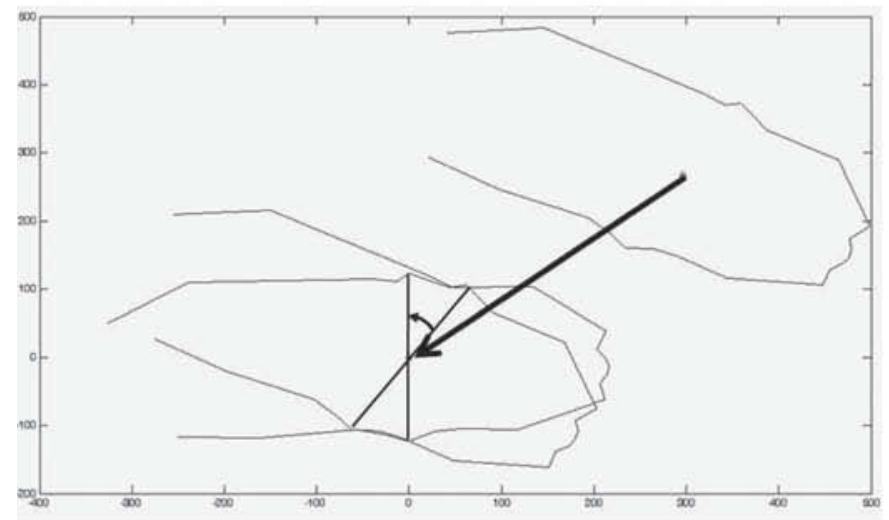

$c$
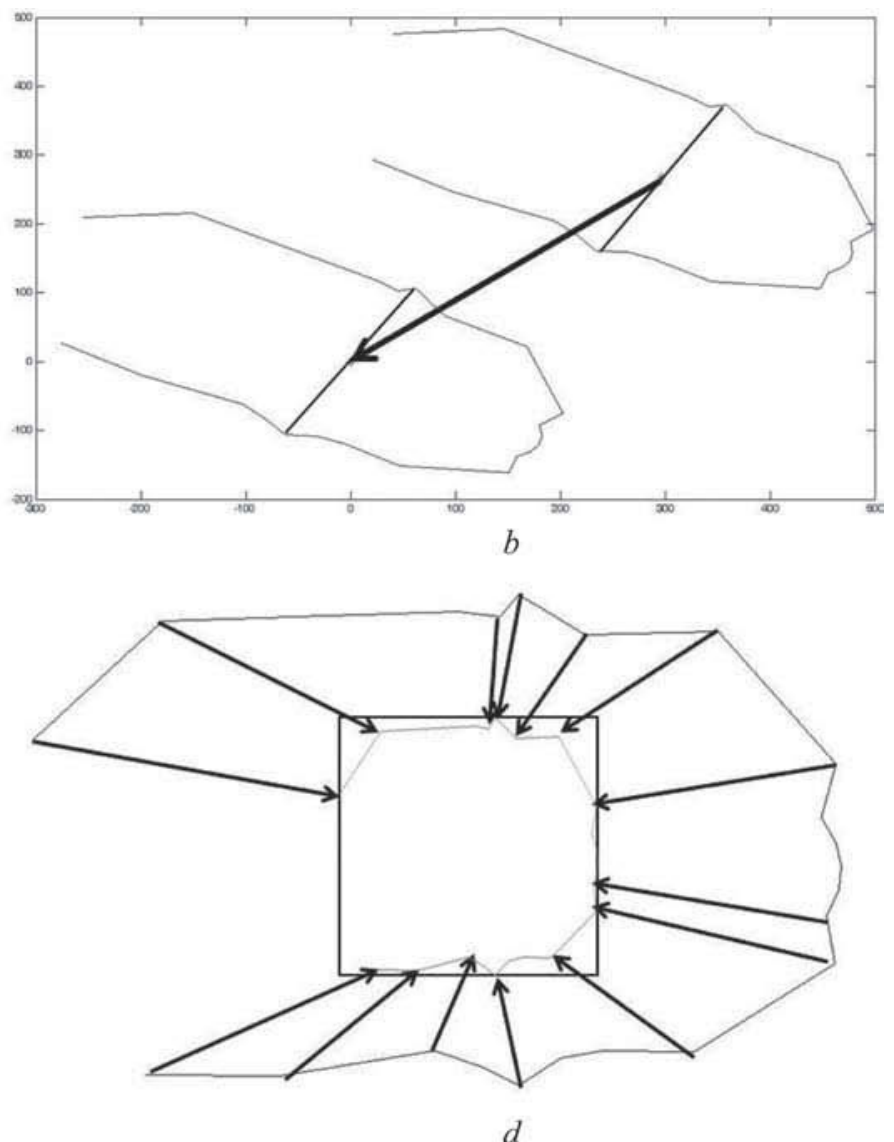

Figure 4. Anatomical landmarks in a cow body shape (a), shape translation (b), shape rotation (c), and shape scaling (d).

PCA is the extension of PCA to deal with nonlinear cases using the technique of kernel methods. The basic idea behind kernel methods is to map the data in the input space ( $\mathrm{S}^{\prime}$ in our case) using a nonlinear function $\Phi$ and then apply a linear method to do further analysis.

Let $\Phi: R^{2 n} \rightarrow R^{n_{\Phi}}$ be a mapping function in the real numbers space acting on the input space $\mathbf{S}^{\prime}$. Kernel PCA finds the principal axes by diagonalizing the covariance matrix $\mathbf{C}_{\Phi}$, built taking into account the samples in $\mathbf{S}_{\Phi}^{\prime}$. The modes of variation into the kernel space are described by the unit eigenvectors of $\mathbf{C}_{\Phi}$, such that

$$
\begin{array}{ll}
\mathbf{C}_{\Phi} \mathrm{e}_{i}^{\Phi}=\lambda_{i}^{\Phi} \mathrm{e}_{i}^{\Phi} & i=1, \ldots, n_{\Phi} \\
\mathrm{e}_{i}^{\Phi T} \mathrm{e}_{i}^{\Phi}=1 & i=1, \ldots, n_{\Phi}
\end{array}
$$

where $\lambda_{i}^{\Phi}$ is the ith eigenvalue of $\mathbf{C}_{\Phi}$. The eigenvectors $\mathrm{e}_{i}^{\Phi}$ of the covariance matrix corresponding to the largest eigenvalues describe the most significant modes of variations in the variables used to derive the covariance matrix $\mathbf{C}_{\Phi}$.
Note that PCA is a particular instance of the kernelized method and the possible mapping function is $\Phi(\mathbf{x})$ $=\mathrm{x}$.

In our experiments, we used kernel PCA to model the shape of cows. Specifically, we used a linear $\left(\Phi_{\text {Linear }}(\mathbf{x})\right.$ $=\mathbf{x}$ for $\left.\mathbf{x}=\left[\mathrm{x}_{1}, \mathrm{x}_{2}\right]\right)$ and a polynomial mapping function $\left(\Phi_{\text {Polynomial }}(\mathbf{x})=\left[x_{1}^{2}, x_{2}^{2}, \sqrt{2 x_{1} x_{2}}\right]\right)$.

\section{Evaluation}

The PCA and kernel PCA-based shape descriptor methods were evaluated by comparing them to other existing methods of BCS scoring using digital images (Bewley et al., 2008 and Halachmi et al., 2008) from the database used in this study.

In the first study, the authors considered a single image of the dorsal view of the cow and used 23 anatomical points to define the shape of the body of the cow. These points, selected in a manual way, were used to compute 15 angles around the hooks, pins, and tailhead. The authors considered the symmetry of the cow shape to obtain 7 composite angles averaging the left and right 
angles of the shape. The related proposed models are reported below:

$$
\begin{gathered}
y_{i j}=\mu+\text { Cow }_{i}+\beta_{1} H A_{i j}+\beta_{2} P H A_{i j} \\
+\beta_{3}(H A \times P H A)_{i j}+e_{i j}(\text { Bewley } 1) \\
y_{i j}=\mu+\text { Cow }_{i}+\beta_{1} H A_{i j}+\beta_{2} P H A_{i j}+\beta_{3}(H A \times P H A)_{i j} \\
+\beta_{4} T D_{i j}+\beta_{5}(P H A \times T D)_{i j}+e_{i j}(\text { Bewley } 2),
\end{gathered}
$$

where $y_{i j}$ is the $j$ th BCS of the $i$ th cow estimated by technicians; $\mu, \beta_{1}, \beta_{2}, \beta_{3}, \beta_{4}$, and $\beta_{5}$ are the regression parameters; $\operatorname{Cow}_{i}$ is the identifier of the $i$ th cow; $e_{i j}$ is the residual error; $H A_{i j}$ is the average hook angle; $P H A_{i j}$ is the average posterior hook angle; and $T D_{i j}$ is the average tailhead depression. In this experiment, based on a training system, the term Cow $_{i}$ in the Bewley 1 and Bewley 2 equations could not be taken into account in testing the models because this value does not provide anatomical information useful for BCS estimation. Technicians do not use the ID of the cow to estimate the BCS and if in the training phase the ID was included in the learning process, the BCS of a new cow (never captured by the system) could not be estimated. Moreover, we think that by knowing the ID of the cow, particularly when BCS is assessed over time, the BCS estimation could be biased from 2 sources, one from the technical observers biased by the prior BCS of a particular cow, and second, by the machine algorithm. Because the machine algorithm learns parameters of the model from BCS, when cow ID are associated a priori with a BCS, changes over time within a cow may be masked by linking BCS with cow ID. Cow BCS must be assigned before linking with cow ID to minimize this bias.

In the second study, the posterior part of the cow was considered and a parabolic fitting was performed. The absolute differences between the real body shape and the fitted parabola were used in the BCS estimation as follows:

$$
B C S_{\text {Thermal }}=5 \times B_{f i t} \times \frac{1}{M A E}(\text { modified Halachmi })
$$

where $B_{f i t}$ is the best fit reached in the herd, $M A E$ is the mean absolute error, and 5 is a normalization factor. In our evaluation phase, we implemented a modified version of Halachmi. Parabolic fitting and BCS estimation were performed considering only the labeled points because we did not use a thermal camera to acquire images and, therefore, shape extraction from those images was a complex task not addressed in this study.

The mean BCS estimation error was defined as:

$$
\overline{\text { BCSError }}=\frac{1}{N} \sum_{i=1}^{N}\left|B C S_{e s t}(i)-B C S_{t e c}(i)\right|
$$

where $N$ is the number of images (i.e., 286), $B C S_{\text {est }}$ and $B C S_{t e c}$ were the BCS values estimated from digital images and the mean BCS manually evaluated by technicians, respectively.

\section{Validation}

To assess the effectiveness of the methods, the leave one out cross validation (LOOCV) procedure (Webb, 2002) and the regression error characteristic curves (REC; Bi and Bennett, 2003) were used. Each run of LOOCV involved a single observation of the data set as test data, and the remaining samples as training data. This was repeated to guarantee that each sample was used once as the test data. The average error rate was computed taking into account all runs. The REC curve is essentially the cumulative distribution function of the error. It was derived by plotting the errors tolerance versus the percentage of samples predicted within the tolerance. The area over the curve is a biased estimation of the expected error of an employed regression model. This technique enabled simple assessment of different

Table 1. Means \pm SD, and range (in parentheses) for milk yield, fat and protein percentage, and BCS

\begin{tabular}{lccccc}
\hline $\begin{array}{l}\text { DIM } \\
\text { class }\end{array}$ & $\mathrm{n}$ & $\begin{array}{c}\text { Milk } \\
(\mathrm{kg})\end{array}$ & $\begin{array}{c}\text { Fat } \\
(\%)\end{array}$ & $\begin{array}{c}\text { Protein } \\
(\%)\end{array}$ & \multicolumn{1}{c}{ BCS } \\
\hline$<60$ & 14 & $24.9 \pm 6.7$ & $3.50 \pm 1.26$ & $3.13 \pm 0.22$ & $2.89 \pm 0.20$ \\
& & $(14.8-34.7)$ & $(2.11-6.99)$ & $(2.90-3.69)$ & $(2.50-3.25)$ \\
$61-120$ & 12 & $30.6 \pm 5.7$ & $3.31 \pm 0.69$ & $3.33 \pm 0.31$ & $3.01 \pm 0.48$ \\
& $(21.0-38.4)$ & $(2.05-4.39)$ & $(2.87-4.10)$ & $(2.25-4.00)$ \\
$121-200$ & 29 & $31.3 \pm 5.5$ & $3.36 \pm 0.64$ & $3.33 \pm 0.27$ & $3.25 \pm 0.55$ \\
& & $(22.6-42.0)$ & $(2.04-4.78)$ & $(2.82-3.83)$ & $(2.50-4.75)$ \\
$>200$ & 19 & $30.2 \pm 4.4$ & $3.41 \pm 0.63$ & $3.42 \pm 0.24$ & $3.49 \pm 0.42$ \\
& & $(24.4-41.1)$ & $(2.50-4.74)$ & $(3.02-3.76)$ & $(3.00-4.25)$ \\
\hline
\end{tabular}


a)

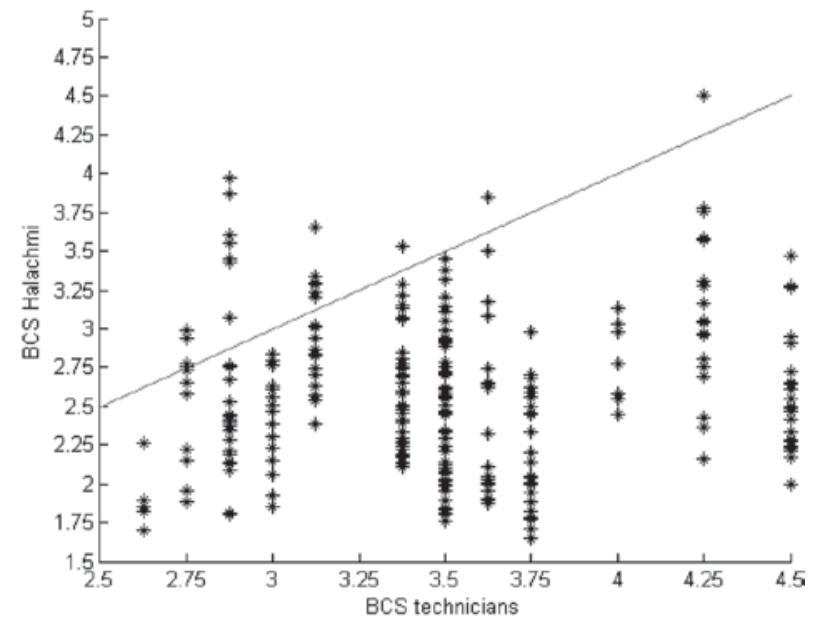

b)

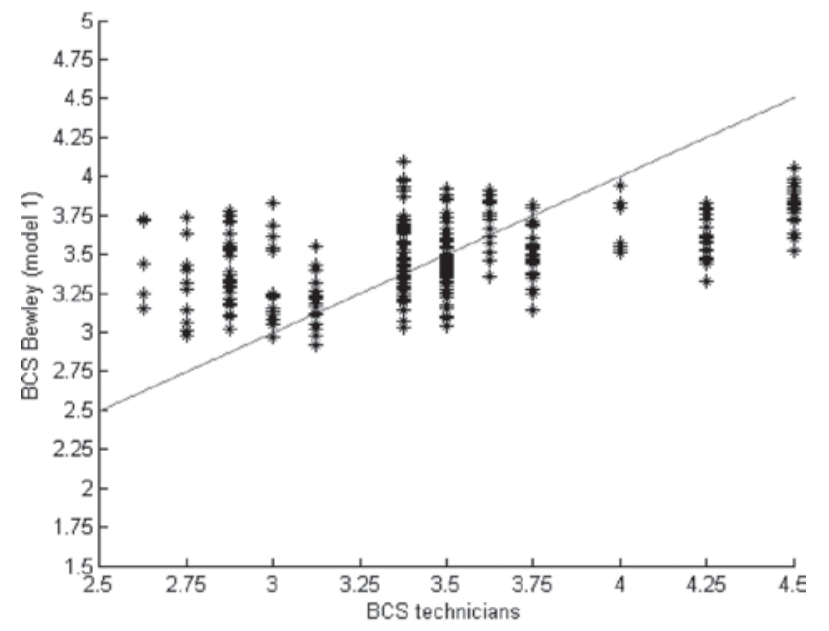

c)

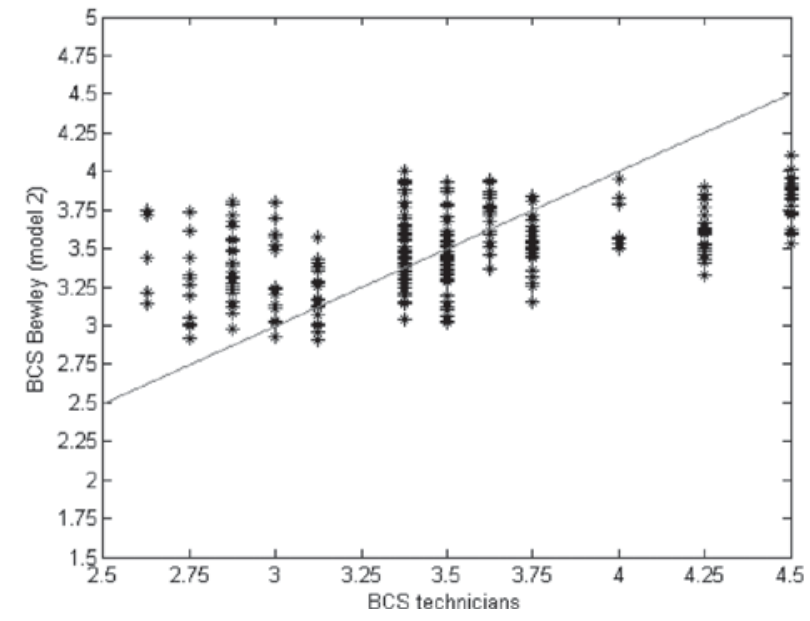

Figure 5. a) Predicted (Halachmi model) versus actual BCS (estimated by technicians). b) Predicted (Bewley model 1) versus actual BCS (estimated by technicians). c) Predicted (Bewley model 2) versus actual BCS (estimated by technicians). a)

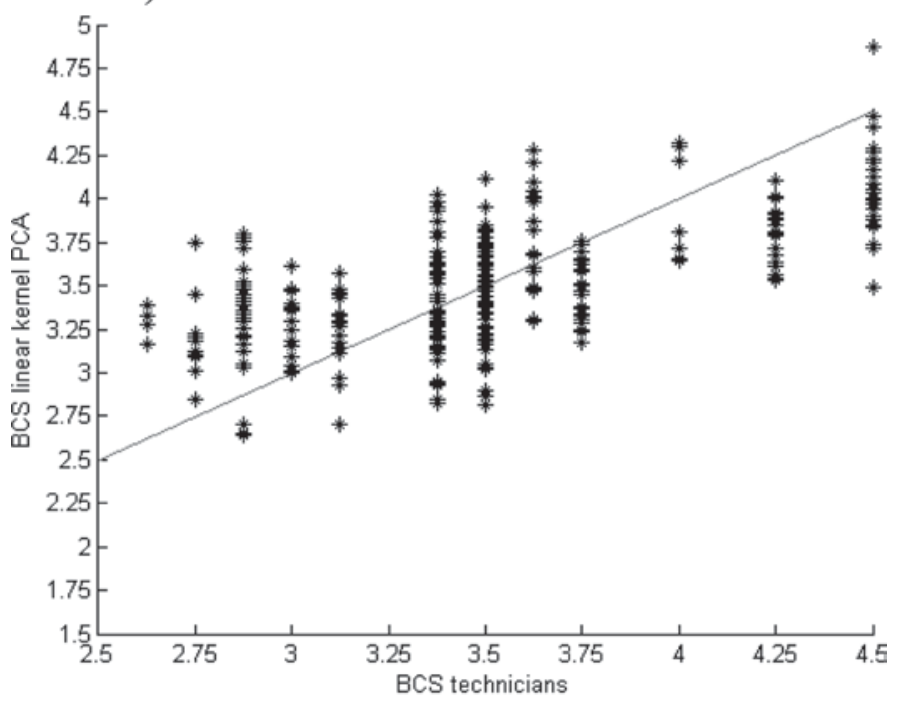

b)

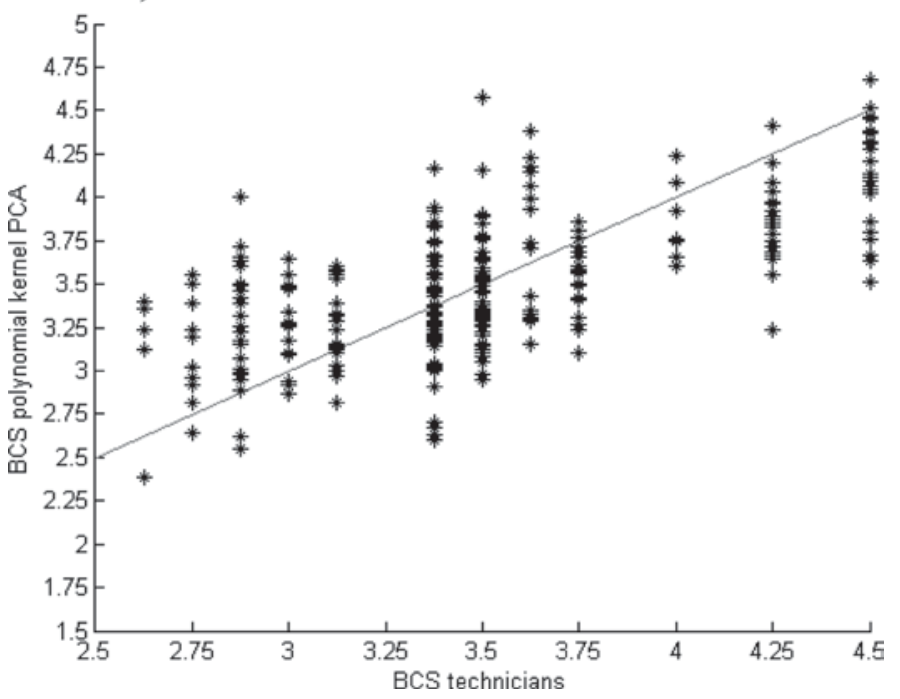

Figure 6. a) Predicted (linear kernel principal component analysis, or PCA) versus actual BCS (estimated by 2 technicians). b) Predicted (polynomial kernel PCA) versus actual BCS (estimated by technicians).

regression models by examining the relative position of their REC curves.

\section{RESULTS AND DISCUSSION}

\section{Data}

During the experiment, 74 first-parity lactating cows were observed (Table 1 ). For each 4 -h acquisition timeframe $(2 \times 26 \mathrm{~d}), 172,800$ images were gathered. The first filtering step using interframe error detected 40 peaks and, for each peak, 200 images were selected. 




Figure 7. Polynomial kernel principal component analysis (PCA) approach (dash-dot line) versus Bewley model 2 (dashed line).

The automatic filtering process led to a final set with 10,400 images. Not all images of an acquisition interval could be used because of blurring and dirtiness due to automatic washing of the barn. The final data set contained, therefore, only 286 manually chosen images, corresponding to 29 different cows. All of the 286 selected images were used in the subsequent analysis and stored together with the related data (anatomical points and BCS) in a database available at http:// www.corfilac.it/bcs/.

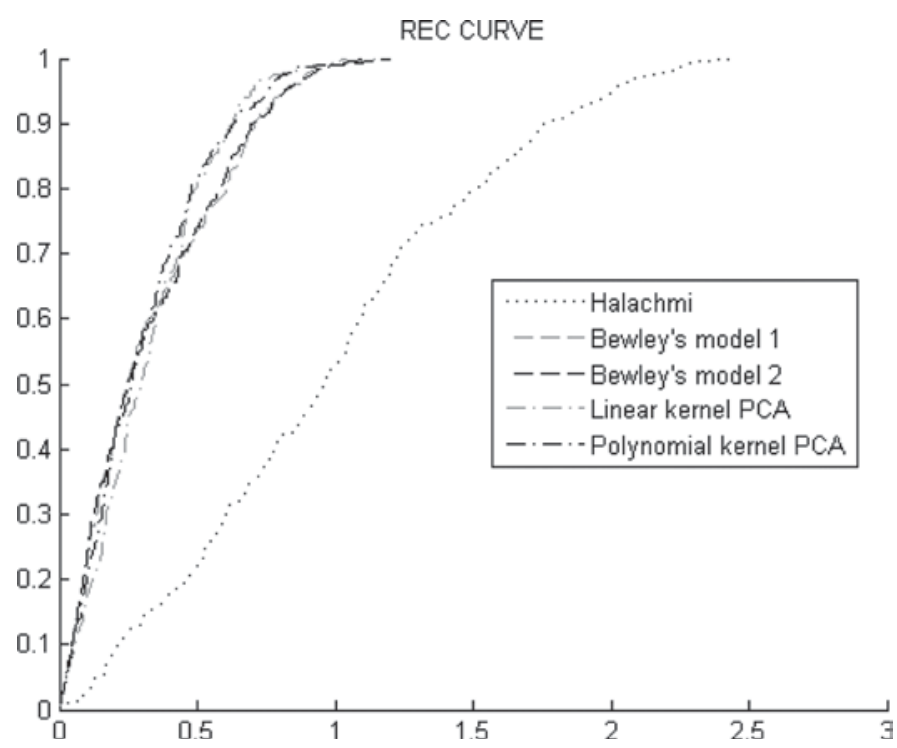

Figure 8. Regression error characteristic curves (REC) of the different models involved in the comparison. $\mathrm{PCA}=$ principal component analysis.

\section{Shape Descriptor}

The eigenvectors $\left\{\mathbf{e}_{i}^{\Phi}\right\}_{i=1}^{n_{\Phi}}$ useful to describe the shapes were computed using kernel PCA. Assuming zero mean, any shape in the training set mapped into the kernel space through $\Phi$ can, therefore, be generated as a linear combination of the eigenvectors obtained through kernel PCA.

This means that the eigenvectors $\left\{\mathbf{e}_{i}^{\Phi}\right\}_{i=1}^{n_{\Phi}}$ constitute a basis of the space of shapes into the kernel space $\Phi\left(\mathbf{S}^{\prime}\right)$ useful to generate new samples. Unseen shapes in the kernel space can be generated by changing the values of each component $a_{i}^{\Phi}$, taking into account that its variance is represented by $\lambda_{i}^{\Phi}$. Because most of the samples of the training set lie within 3 standard deviations of the mean, the suitable range for $a_{i}^{\Phi}$ is $\left[-3 \sqrt{\lambda_{i}^{\Phi}}, 3 \sqrt{\lambda_{i}^{\Phi}}\right]$. The range of each $a_{i}^{\Phi}$ can be used to detect outliers that, in our case, are probably due to error in manual labeling.

Given a training set of cow shapes, kernel PCA can be applied after alignment and, hence, each shape $\mathbf{s}_{j}^{\prime}$ can be described by using the vector $\mathbf{a}_{j}^{\Phi}=\left[a_{j, 1}^{\Phi}, \ldots, a_{j, n_{\Phi}}^{\Phi}\right]$.

The shape descriptors of the training set can be used together with a linear regressor to build a system for BCS estimation:

$$
B C S_{j}=a_{j, n_{\Phi}}^{\Phi} w_{n_{\Phi}}+a_{j, n_{\Phi}-1}^{\Phi} w_{n_{\Phi}-1}+\ldots+a_{j, 1}^{\Phi} w_{1}+w_{0} .
$$

Given the descriptors of the shapes in the training set, the regression model can be fitted by using a least squares method. The learned parameters $\left[w_{0}, w_{1}, \ldots, w_{n_{\Phi}}\right]$ are then used to infer the BCS of new shape samples, describing them by using the basis $\left[\mathbf{e}_{1}^{\Phi}, \ldots, \mathbf{e}_{n_{\Phi}}^{\Phi}\right]$ learned on the training set.

\section{Validation}

In the validation step, BCS was estimated using the 4 different models over the images of the training set and errors were averaged for all runs of LOOCV. Among the methods, the modified Halachmi method reported the biggest error (0.98), whereas errors for both Bewley 1 and Bewley 2 models and our linear and polynomial kernel PCA were $0.33,0.32$, and 0.31 respectively. The modified Halachmi approach was not able to provide satisfactory results (Figure 5a): the parabolic fitting might be not accurate enough because it was performed 
considering only the labeled points. Bewley 1 and Bewley 2 models obtained similar results (model 2 was slightly better than the model 1; Figure 5b and Figure 5c). Their performances are better for the central BCS values (around 3.5) and worst in the extreme cases (2.5 and 4.5). Our approach, in particular the polynomial one, performed better than the other techniques, obtaining satisfactory results even in the extreme cases. Results of our approach are reported in Figure 6a and Figure 6b. As shown in Figure 7, our approach using a polynomial kernel is able to follow the ideal line better than do the Bewley 1 and Bewley 2 approaches. In Figure 8 the comparison through the REC curve confirms that the proposed approach performs better than do state-of-the-art methods in estimating BCS.

\section{CONCLUSIONS}

The cow body shape was described, considering the deviation from the average shape in the kernel space. The method produced a description of the shape to be used for automatic estimation of BCS. Experimental results confirm the effectiveness of the proposed approach. Body condition score estimation systems that work fully automatically (with no user intervention) or at least semiautomatically (with minimal user intervention) are desired in order to cut down time and costs of the traditional BCS estimation techniques. Moreover, these systems can produce an objective evaluation of BCS in a way that is less invasive for the cows. The main drawback of a semiautomatic system for BCS estimation is the manual labeling of anatomical points during training and employing phases. Future work will be devoted in building a fully automatic system for BCS evaluation in which the shape of a cow will be automatically extracted from digital images acquired with a low-cost camera through a segmentation procedure. The automatic extraction of the whole shape could be also useful to build a more robust model for BCS estimation by using more anatomical points, automatically extracted. Finally, a benchmark data set useful for dairy cattle research purposes, available through the Internet, was built.

\section{ACKNOWLEDGMENTS}

Financial support was provided by the Assessorato Agricoltura e Foreste della Regione Siciliana (Palermo, Italy).

\section{REFERENCES}

Agnew, R. E., and T. Yan. 2000. Impact of recent research on energy feeding systems for dairy cattle. Livest. Prod. Sci. 66:197-215.
Bastin, C., S. Loker, N. Gengler, A. Sewalem, and F. Miglior. 2010. Genetic relationships between body condition score and reproduction traits in Canadian Holstein and Ayrshire first-parity cows. J. Dairy Sci. 93:2215-2228.

Belongie, S., J. Malik, and J. Puzicha. 2002. Shape matching and object recognition using shape contexts. IEEE Trans. Pattern Anal. Mach. Intell. 24:509-522.

Berry, D. P., F. Buckley, P. Dillon, R. D. Evans, M. Rath, and R. F. Veerkamp. 2003. Genetic relationships among body condition score, body weight, milk yield, and fertility in dairy cows. J. Dairy Sci. 86:2193-2204.

Bewley, J. M., A. M. Peacock, O. Lewis, R. E. Boyce, D. J. Roberts, M. P. Coffey, S. J. Kenyon, and M. M. Schutz. 2008. Potential for estimation of body condition scores in dairy cattle from digital images. J. Dairy Sci. 91:3439-3453.

Bi, J., and K. P. Bennett. 2003. Regression error characteristic curves. Proc. 20th Int. Conf. Machine Learning, Washington DC. The AAAI Press, Menlo Park, CA.

Coffey, M. P., N. McFarlane, and T. Mottram. 2003. The feasibility of automatic condition scoring. Holstein J. 66:82-83.

Cootes, T. F., G. J. Edwards, and C. J. Taylor. 2001. Active appearance models. IEEE Trans. Pattern Anal. Mach. Intell. 23:681685.

Cootes, T. F., C. J. Taylor, D. H. Cooper, and J. Graham. 1992. Training models of shape from sets of examples, Proc. British Machine Vision Conference (Berlin), Springer, pp. 266-275.

Dechow, C. D., G. W. Rogers, and J. S. Clay. 2002. Heritability and correlations among body condition score loss, body condition score, production and reproductive performance. J. Dairy Sci. $85: 3062-3070$.

Di Fabio, B., C. Landi, and F. Medri. 2009. Recognition of occluded shapes using size functions. Proc. 15th Int. Conf. Image Anal. Processing. Lecture Notes in Computer Science. 5716:642-651.

Domecq, J. J., A. L. Skidmore, J. W. Lloyd, and J. B. Kaneene. 1997a. Relationship between body condition scores and conception at first artificial insemination in a large dairy herd of high yielding Holstein cows. J. Dairy Sci. 80:113-120.

Domecq, J. J., A. L. Skidmore, J. W. Lloyd, and J. B. Kaneene. 1997b. Relationship between body condition scores and milk yield in a large dairy herd of high yielding Holstein cows. J. Dairy Sci. 80:101-112.

Dryden, I. L., and K. V. Mardia. 1998. Statistical Shape Analysis. John Wiley \& Sons, Inc., New York, NY.

Edmonson, A. J., I. J. Lean, L. D. Weaver, T. Farver, and G. Webster. 1989. A body condition scoring chart for Holstein dairy cows. J. Dairy Sci. 72:68-78.

Ferguson, J. D., G. Azzaro, and G. Licitra. 2006. Body condition assessment using digital images. J. Dairy Sci. 89:3833-3841.

Ferguson, J. D., D. T. Galligan, and N. Thomsen. 1994. Principal descriptors of body condition in Holstein dairy cattle. J. Dairy Sci. 77:2695-2703.

Gonzalez, R. C., and R. E. Woods. 2008. Digital Image Processing. 3rd ed. Prentice-Hall, Upper Saddle River, NJ.

Hady, P. J., J. J. Domecq, and J. B. Kaneene. 1994. Frequency and precision of body condition scoring in dairy cattle. J. Dairy Sci. 77:1543-1547.

Halachmi, I., P. Polak, D. J. Roberts, and M. Klopcic. 2008. Cow body shape and automation of condition scoring. J. Dairy Sci. 91:4444-4451.

Otto, K. L., J. D. Ferguson, D. G. Fox, and C. J. Sniffen. 1991. Relationship between body condition score and composition of the ninth to eleventh rib tissue in Holstein dairy cows. J. Dairy Sci. 74:852-859.

Persoon, E., and K.-S. Fu. 1977. Shape discrimination using Fourier descriptors. IEEE Trans. Syst. Man. Cybern. 7:170-179.

Pryce, J. E., M. P. Coffey, and S. Brotherstone. 2000. The genetic relationship between calving interval, body condition score and linear type and management traits in registered Holsteins. J. Dairy Sci. 83:2664-2671.

Pryce, J. E., M. P. Coffey, S. H. Brotherstone, and J. A. Woolliams. 2002. Genetic relationships between calving interval and body 
condition score conditional on milk yield. J. Dairy Sci. 85:15901595.

Rathi, Y., S. Dambreville, and A. Tannenbaum. 2006. Statistical Shape Analysis using Kernel PCA. Image Processing: Algorithms and Systems, Neural Networks, and Machine Learning. Proc. SPIE, Vol. 6064, 60641B. E. R. Dougherty, J. T. Astola, K. O. Egiazarian, N. M. Nasrabadi, and S. A. Rizvi, ed. Georgia Institute of Technology, Society of Photo-Optical Instrumentation Engineers, Atlanta, GA.

Sahbi, H. 2007. Kernel PCA for similarity invariant shape recognition. Neurocomputing 70:3034-3045.

Stegmann, M. B., and D. D. Gomez. 2002. A brief introduction to statistical shape analysis. Informatics and Mathematical Modelling, Technical University of Denmark, Lyngby.
Wall, E., S. Brotherstone, J. A. Woolliams, G. Banos, and M. P. Coffey. 2003. Genetic evaluation of fertility using direct and correlated traits. J. Dairy Sci. 86:4093-4102.

Ward, W. R. 2003. Body condition scoring-Technique and application. Cattle Pract. 11:111-116.

Webb, A. R. 2002. Statistical Pattern Recognition. 2nd ed. John Wiley \& Sons, Ltd., West Sussex, UK.

Wildman, E. E., G. M. Jones, P. E. Wagner, R. L. Boman, H. F. Troutt Jr., and T. N. Lesch. 1982. A dairy cow body condition scoring system and its relationship to selected production characteristics. J. Dairy Sci. 65:495-501. 